\title{
Application of Finite Element Method in Large-span Beam Bridge
}

\author{
Ding Wenxia, Guo Qinghua \\ Hubei Communications Technical College
}

Keywords: Large-span overpass, finite element analysis, elastic dynamic, dynamic test, model updating.

\begin{abstract}
In the analysis of large structures, usually finite element model according to the blueprint, the finite element model thus created often fail to reflect the true state of the actual structure. It is necessary to establish the finite element model is corrected. Typically, finite element model updating is defined as a rigidity and quality test results of the analysis of dynamic frequency, mode shape modification theory of finite element model parameters to ensure the accuracy of modal parameters premise itself, so that the vibration parameters finite element model corrected tended test values. After finite element model updating can be applied to structural dynamic response and dynamic security analysis again, some vibration control technology control strategy formulation, structural damage identification, the bridge health monitoring and safety assessment, structural optimization design and the like. This paper evaluates the structural model features required by the state of the bridge structure, modeling methods are given to build large span bridges for structural condition assessment model for the bridge structure such as pylon, deck lines and coupling conditions, etc. Finite element simulation and thus to assess the structural state of the bridge.
\end{abstract}

\section{Introduction}

With the cable-stayed span overpass increasing gradually formed a large-span overpass, and the stability of large-span overpass has been the focus of our research in the overall structure of the size of the bridge for bridge vibration response significant role. We used finite element analysis of large-span overpass analysed. usually large-span cable-stayed overpass main tower pier, pier, four-part main beam and stay cables. Its main bridge pier has a supporting role, it is subjected to a load is small, but its has good stability, so we do finite element analysis. Main Tower Pier is a core component of our load, we use the main pier rod element simulation tower architecture. There are two main girder beam bridge across the ribs, intercostal girder deck surface and cross divisions. The cable-stayed only in tension and not under pressure. To study the mechanical properties of a bridge engineering has become the consensus of the finite element model of the bridge structure to establish a large cross. Existing mature classic bridge models are mainly: Single-girder model, Double girder model, Triple girder model and other models to study these bridges modal characteristics as well as the new highspan bridge design and construction played a key effect. 


\section{Finite Element Model}

(1) Single girder model

Single girder deck system with a single model for the beam to be replaced by an equivalent crosssectional nature of the deck gives way to the beam, deck and the cable connection arm just by asking. As shown in Figure 1.

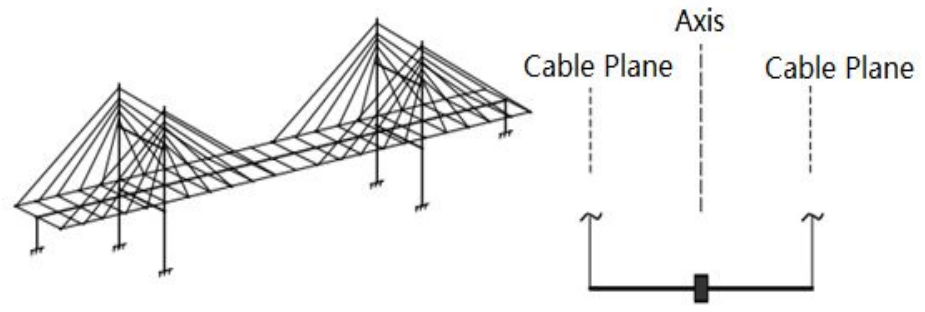

Figure 1.single girder model

12 DOF single girder beam element model for the equivalent beam elements with the classic, on a common commercial software can be easily realized. However, this model is difficult to simulate the deck warping rigidity, therefore suitable only for pure simulation with a larger torsional stiffness Floor System (this time warping stiffness is negligible). For a cross-section of the bridge opening, the bridge of pure torsional stiffness is small, warping stiffness in modal analysis significantly affected, with a single main beam model to simulate the deck department will bring large error.

(2) Double main beam model

Double main beam model consists of two main beam equivalent composition, asked in via the crossbar. Q. distance from the main beam to take two cable plane, the beams ask to take away from the cable. This model cross-sectional area of each piece of the main beam and vertical bending stiffness were taken $1 / 2$ full - section values, using lateral rigidity equal deflection principle equivalent calculations. Deck warping stiffness can be easily negated by two vertical bending stiffness of the main beam obtained when beam is taken as just arm (rigid), the vertical stiffness of the bridge deck will also pass through two main vertical beam rigidity reflected, which will increase the deck equivalent vertical stiffness, rigidity and the lateral rigidity warping calculation uncertainty. When the beam is taken as an elastic beam, the beam cross-sectional nature of the allocated portion of the deck, which will greatly increase the computational equivalent of vertical, lateral and torsional stiffness of the workload, and this time double main beam model in a transverse shear mode structure, mechanical properties significantly distorted the original bridge deck system, resulting in lateral rigidity sectional distortion. These factors make it difficult to double main beam model easily applied to simulate the actual bridge structure. As shown in Figure 2.

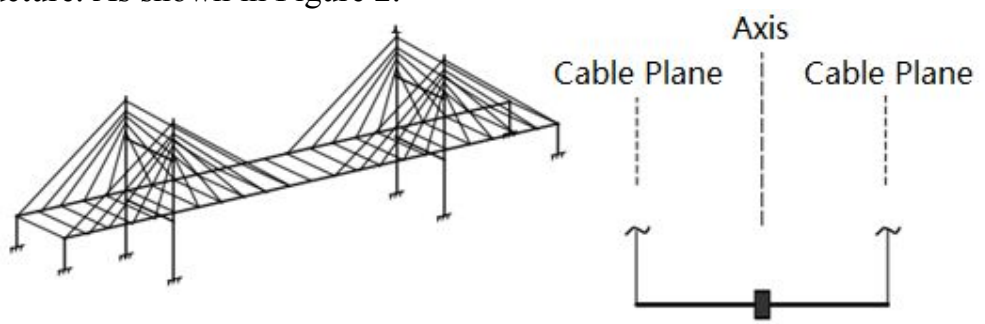

Figure 2. Double main beam model

(3) Three main beam model

Three main beam model consists of two identical bridge is located in the central axis of the beam and the surface of the cable at the side beam composition, asked the three-piece main beam by just connecting arm, wherein the arm rotation is not just about the constraints on both sides of the beam axis of rotation white by the degree that both sides of the beam is rotated around the axis of rotation by a white whiteness and the degree of the main beam is independent. 
Three main beam model can be easily simulated deck warping stiffness, significantly improves the torsional rigidity of pure simulation accuracy smaller opening cross-section in the form of bridge lines for bridges torsional frequency precision computing has made important contributions. But three main beam model. Pure torsional stiffness of the bridge deck can be assigned to any of the three main beams, which makes the process of calculating the equivalent of great uncertainty. As shown in Figure 3.

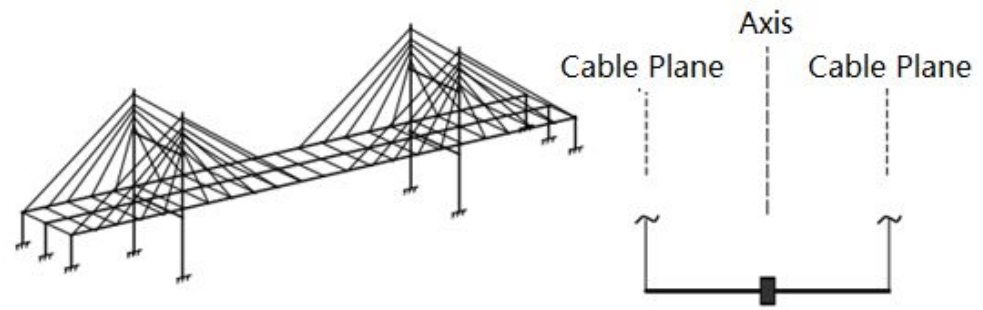

Figure 3. Three main beam model

Therefore, finite element simulation, we only use its pull rod element, finite element simulation, finite element model of its large-span cable-stayed bridge analysis, shown in Figure 4, Material parameters are shown in Table 1.
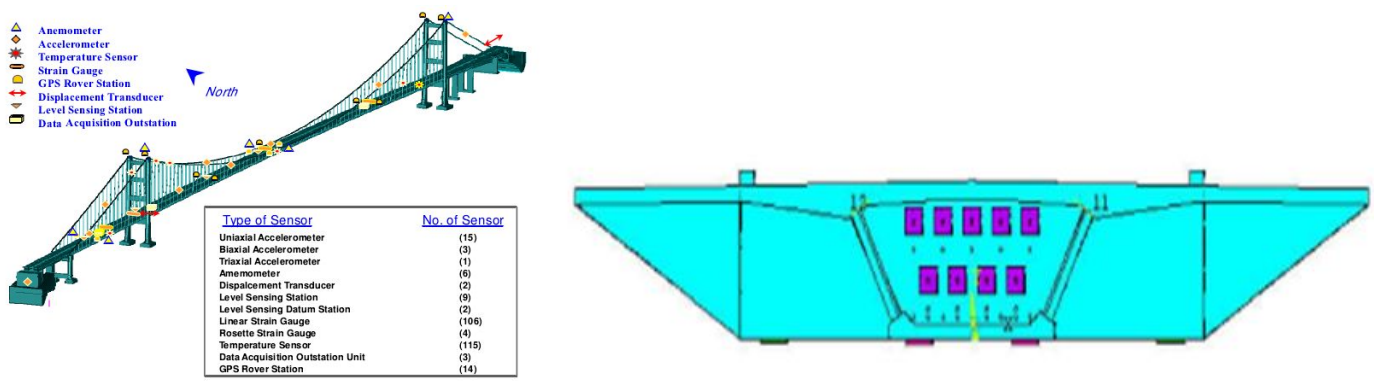

Figure 4. Finite element analysis model

Table 1. Material parameters

\begin{tabular}{|c|c|c|c|c|c|c|c|c|}
\hline & \multicolumn{4}{|c|}{ Material parameters } & \multicolumn{3}{c|}{ Sectional parameters } \\
\cline { 2 - 8 } member & $\begin{array}{c}\text { Elastic } \\
\text { Modulus } \\
(\mathrm{GPa})\end{array}$ & $\begin{array}{c}\text { Poisson' } \\
\text { s ratio }\end{array}$ & $\begin{array}{c}\text { Shear } \\
\text { modulus } \\
(\mathrm{GPa})\end{array}$ & $\begin{array}{c}\text { Density } \\
(\mathrm{kg} / \mathrm{m} 3)\end{array}$ & $\begin{array}{c}\text { area } \\
(\mathrm{m} 2)\end{array}$ & $\begin{array}{c}\text { Moment } \\
\text { of inertia } \\
\mathrm{I} 11 \\
(\mathrm{~m} 4)\end{array}$ & $\begin{array}{c}\text { Moment } \\
\text { of inertia } \\
\text { I22 } \\
(\mathrm{m} 4)\end{array}$ & $\begin{array}{c}\text { Polar } \\
\text { moment of } \\
\text { inertia } \\
(\mathrm{m} 4)\end{array}$ \\
\hline $\mathrm{CM}$ & 196 & 0.3 & 75.395 & 7935 & 0.7593 & $4.588 \mathrm{E}-2$ & $4.588 \mathrm{E}-2$ & $9.175 \mathrm{E}-2$ \\
\hline $\mathrm{CS}$ & 196 & 0.3 & 75.395 & 7935 & 0.8007 & $5.102 \mathrm{E}-2$ & $5.102 \mathrm{E}-2$ & $1.0204 \mathrm{E}-1$ \\
\hline $\mathrm{H}$ & 200 & 0.3 & 76.923 & 7800 & $1.815 \mathrm{E}-2$ & $1.45 \mathrm{E}-3$ & $1.45 \mathrm{E}-3$ & $6.3303 \mathrm{E}-5$ \\
\hline
\end{tabular}

\section{Mathematical Model}

First, we establish the basic model according to its dynamics elastic elastic deformation, the basic equations of the elastic dynamics are as follows: 
(1)The equations of motion

$$
\sigma_{i j, j}+\sigma f_{i}=\rho i j
$$

(2)The geometric equation

$$
\varepsilon_{i j}=\frac{1}{2}\left(u_{i, j}+u_{j, i}\right)
$$

(3) Physical equations

$$
\left\{\begin{array}{l}
\sigma i_{j}=\lambda \varepsilon_{K K} \delta_{i j}+\mu \varepsilon_{i j} \\
\sigma_{K K}=\lambda+\mu \varepsilon_{K K}=K \varepsilon_{K K}
\end{array}\right.
$$

(4) The boundary conditions

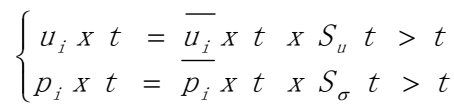

In this function, boundary conditions, there are, and we are $p_{i}=\sigma_{i j} n_{j}$, $S_{u}+S_{\sigma}=s, \overline{u_{i}}(x, t)$ and $\overline{p_{i}}(x, t)$ is the previously calculated function values obtained. We Elastodynamics model of large-span overpass, we must first establish the Pan function, usually using the variational principle to the establishment of the Panfunction basis Elastodynamics numerical methods, by their stationary condition can be obtained elastic dynamics equations and boundary conditions.

\section{3 structural analysis model}

(1) stability calculations

After our analysis and numerical simulation of linear, nonlinear We obtain a large span of the bridge buckling mode of deformation and stress conditions. The main part of the deformation is the main beam, shown in Figure 4.

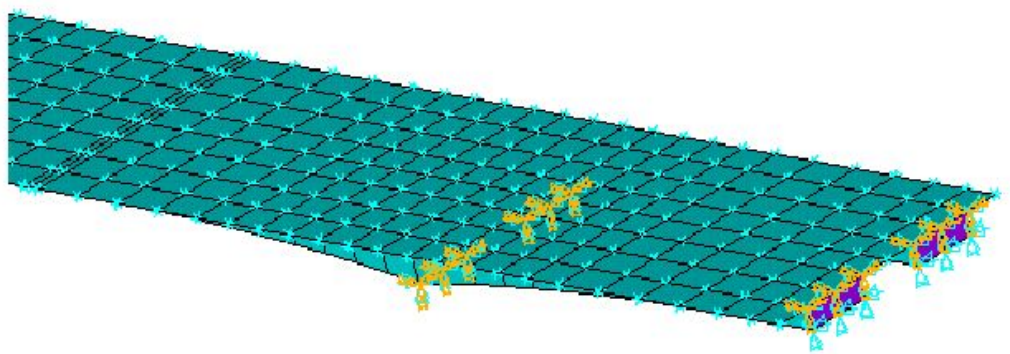

Figure 5. Main beam model

(2) dynamic vibration chatter stability response process

The entire process of dynamic response of the bridge consists mainly elastic deformation of the three phases of construction, local plastic deformation phase structure, phase stability undermine the structure. As shown in Figure 6. 


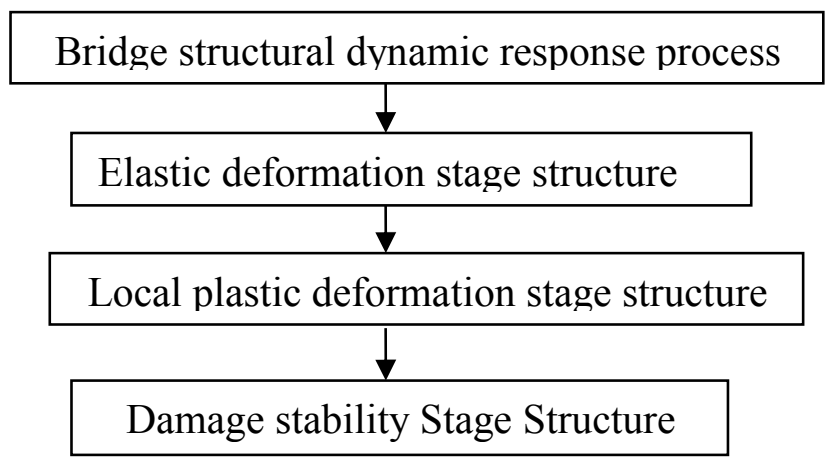

Figure 6. Dynamic Response of Bridges

(1)Elastic Stage Structure:When the entire bridge shaped structure in elastic stage, nodal displacements and axial force in response to the node is the main factor to consider balanced.

(2)Structure of local plastic deformation phases:Axis force local plastic deformation stage at the support structure of the distribution of stress is the main structure of the local plastic deformation phase. When the elastic deformation of the local shaping stage, due to the lever residual deformation leading to structural equilibrium position changing. Cause plastic deformation.

(3)Stability of the structure damage stage:Elastic deformation in both stages of the destruction of the stability of the structure, a further plastic deformation. The plastic deformation is the main factor causing damage to the stability of the structure.

Because when the bridge receives a larger load, displacement exhibits a nonlinear relationship, there is no scaling properties. When you receive a large load carrying capacity of the shaft at the support force is applied to gauge emerged maximum. When the bridge structure into the elastoplastic stage, residual deformation of the lever, the equilibrium position is constantly changing structure, vibration beam bridge continue in the new equilibrium position, this response was mainly due to plastic deformation caused by the lever.

\section{Conclusion}

Through the analysis we get the eigenvalues structural dynamics, we can see through the eigenvalues in the construction process and the state of its bridges are generally in greater linear stability factor of safety. Research work of this paper is to address the needs of large-span steel bridge structure health monitoring and condition assessment of the operation, around span bridges finite element model modeling strategy, model updating theory, model validation and fatigue damage analysis and expand, its purpose for the development and numerical modeling for large span bridges condition assessment of computing technology. The main work done by this and this article summarizes the main results achieved, and the subsequent work prospects. 


\section{References}

1. S.W.Doebling, C.R.Farrar. Computation of structural flexibility for bridge health monitoring using ambient modal data. In roceedings of the $11^{\text {th }}$ ASCE Engineering MechaniCS conference, 2010, 1114-1117

2. M.A. Green: High Efficiency Silicon Solar Cells (Trans Tech Publications), Switzerland 2007.

3. Y. Mishing, in: Diffusion Processes in Advanced Technological Materials, edtied by D. Gupta Noyes Publications/William Andrew Publising, Norwich, NY (2014).

4. G. Henkelman, G.Johannesson and H. Jónsson, in: Theoretical Methods in Condencsed Phase Chemistry, edited by S.D. Schwartz, volume 5 of Progress in Theoretical Chemistry and Physics, chapter, 10, Kluwer Academic Publishers (2000).

5. R.D.Cook concept and application of finite element analysis. Beijing: Science Press, 2009.1-9

6. Wang break into, Shao Min finite element method and the basic principles of numerical methods. Beijing: Tsinghua University Press, 2007,1-2.

7. Du Qing, Zhang Xianmin. Bridges and other reinforced concrete structures dynamic finite element model updating Highway and Transportation Research, 2006, 23 (1): 60-63

8. Wei Afterlife Review correction method finite element dynamic model of Vibration and Shock, 2008, 17 (3): 43-46

9. Yong Cao, Xiaoguang Yue, Fei Xiong and Youjie Zhao.The Software Reliability Model Using Hybrid Model of Fractals and BP Neural Network. IETI Transactions on Computers. 2015.1(1): :11-21

10. Mottershead J E. Friswel ML. Model updating in structural dynamics: A Survey. Sound and Vibration, 1993,167:347-375

11. Gyemng-Ho Kim, Yotm-Sik Park. An improved updating parameter selection method and finite element model updating using multi objective optimization technique. Mechanical Systems and Signal Processing, 12-16 